1995

\title{
Portfolio Inefficiency and the Cross-Section of Expected Returns
}

Shmuel Kandel

Robert F. Stambaugh

University of Pennsylvania

Follow this and additional works at: https://repository.upenn.edu/fnce_papers

Part of the Finance Commons, and the Finance and Financial Management Commons

\section{Recommended Citation}

Kandel, S., \& Stambaugh, R. F. (1995). Portfolio Inefficiency and the Cross-Section of Expected Returns. The Journal of Finance, 50 (1), 157-184. http://dx.doi.org/10.1111/j.1540-6261.1995.tb05170.x 


\title{
Portfolio Inefficiency and the Cross-Section of Expected Returns
}

\begin{abstract}
The Capital Asset Pricing Model implies that (i) the market portfolio is efficient and (ii) expected returns are linearly related to betas. Many do not view these implications as separate, since either implies the other, but we demonstrate that either can hold nearly perfectly while the other fails grossly. If the index portfolio is inefficient, then the coefficients and from an ordinary least squares regression of expected returns on betas can equal essentially any values and bear no relation to the index portfolio's meanvariance location. That location does determine the outcome of a mean-beta regression fitted by generalized least squares.
\end{abstract}

Disciplines

Finance | Finance and Financial Management 
NBER WORKING PAPER SERIES

\section{PORTFOLIO INEFFICIENCY AND THE CROSS-SECTION OF EXPECTED RETURNS}

Shmuel Kandel

Robert F. Stambaugh

Working Paper No. 4702

\section{NATIONAL BUREAU OF ECONOMIC RESEARCH 1050 Massachusetts Avenue \\ Cambridge, MA 02138 \\ April 1994}

The authors are grateful for comments by Yakov Amihud, Ravi Jagannathan, Simon Wheatley, an anonymous referee, and workshop participants at the University of Pennsylvania. This paper is part of the NBER's research program in Asset Pricing. Any opinions expressed are those of the authors and not those of the National Bureau of Economic Research. 


\title{
PORTFOLIO INEFFICIENCY \\ AND THE CROSS-SECTION OF \\ EXPECTED RETURNS
}

\begin{abstract}
A plot of expected returns versus betas obeys virtually no relation to an inefficient index portfolio's mean-variance location. If the index portfolio is inefficient, then the coefficients and $R$-squared from an ordinary-least-squares regression of expected retums on betas can equal essentially any desired values. The mean-variance location of the index does determine the properties of a cross-sectional mean-beta relation fitted by generalized least squares (GLS). As the index portfolio moves closer to exact efficiency, the GLS mean-beta relation moves closer to the exact linear relation corresponding to an efficient portfolio with the same variance. The goodness-of-fit for the GLS regression is the index portfolio's squared relative efficiency, which measures closeness to efficiency in mean-variance space.
\end{abstract}

Shmuel Kandel

Finance Department

The Wharton School

University of Pennsylvania

Philadelphia, PA 19104-6367
Robert F. Stambaugh

Finance Department

The Wharton School

University of Pennsylvania

Philadelphia, PA 19104-6367

and NBER 


\section{Introduction}

Expected returns on a set of risky assets obey an exact linear relation to betas computed against an index portfolio that lies on the minimum-variance boundary of those assets. If the betas are computed instead against an index portfolio that lies inside the minimum-variance boundary, then expected returns must deviate to some degree from any fitted cross-sectional linear relation. ${ }^{1}$ These properties are well known, but they leave open the question of whether, in the latter case, the extent to which expected returns are approximated by a linear function of beta is at all related to the mean-variance location of the index portfolio. For example, one might ask whether, with only negligible inefficiency in the index portfolio, a plot of expected returns versus betas would display a near-perfect linear relation.

In fact, the mean-variance location of an inefficient index portfolio bears essentially no relation to the plot of expected returns versus betas. For example, expected returns can display essentially no correlation with betas computed against an index portfolio that has an expected return arbitrarily close to that of the efficient portfolio with the same variance. Alternatively, expected returns can display a nearly perfectly linear relation to betas computed against an index portfolio that is grossly inefficient. Such plots of expected returns versus betas can be summarized by ordinary least squares (OLS) regression. We show that, if the index portfolio is inefficient, the OLS regression coefficients and R-squared can equal essentially any values desired. This general result, as well as the two examples noted above, can be demonstrated by repackaging a given set of risky assets into alternative sets that generate the same portfolio opportunities. Such repackagings change neither the index portfolio nor the minimum-variance boundary, but they can change the cross-sectional mean-beta relation in virtually any manner desired.

This study shows that generalized-least-squares (GLS) regression provides a framework wherein the exact linear mean-beta relation implied by strict efficiency of the index portfolio can be generalized to an approximate linear relation when the index is inefficient. The GLS regression uses the covariance matrix of the asset returns, and much of the information in that matrix is omitted in a plot of expected return versus beta. An index portfolio's location in mean-variance space is unaffected by repackaging the individual assets, and we define a measure of relative efficiency that is determined by a portfolio's mean-variance location. This relative-efficiency measure approaches its maximum value of unity as the index portfolio moves closer to the upper portion of the minimum-variance boundary. We find that this measure provides a simple link between the index portfolio's mean-variance

\footnotetext{
'See, Fama (1976), Roll (1977) and Ross (1977).
} 
location and the properties of the fitted GLS mean-beta relation. As the index portfolio's relative efficiency moves closer to unity, the fitted GLS mean-beta relation moves closer to the exact linear relation corresponding to an efficient portfolio with the same variance as the index. A slope coefficient of zero occurs only when the mean return on the index is equal to that of the global minimum-variance portfolio. Moreover, the goodness-of-fit measure for the GLS cross-sectional regression is simply the squared relative efficiency of the index portfolio.

In the absence of an exact linear relation between expected returns and betas, a variety of criteria could be used to fit a line and judge its goodness-of-fit. Developing such criteria is difficult without an economic context in which to view a fitted linear mean-beta relation. We consider a context in which such a relation is judged by its ability to provide fitted expected returns that are useful substitutes for true expected returns as inputs to a standard one-period portfolio optimization. For a given set of cross-sectional independent variables, including but not limited to beta, using the expected returns fitted from a GLS. regression produces a portfolio with a higher expected return than using any other linear function of the independent variables. The squared relative efficiency of that portfolio is simply the goodness-of-fit for the GLS regression.

The analysis in this study takes the moments of the return distribution as given, so sampling issues of estimation and inference are not addressed. Such issues are beyond the intended scope of this paper. For an analysis of these issues as they apply to the GLS crosssectional regression, the interested reader is referred to Shanken (1992). He shows that, when sample mean returns are regressed on estimated betas, the GLS estimator is asymptotically efficient, even though the betas used as independent variables are first estimated in OLS regressions. ${ }^{2}$ Shanken also observes, however, that there are reasons to be concerned about the small-sample performance of the GLS estimator. In recent empirical work, Amihud, Christensen, and Mendelson (1992) report that a GLS regression produces a significantly positive slope for the mean-beta relation, in contrast to the OLS result reported by Fama and French (1992). For an analysis of how the sample mean-variance location of the index portfolio is related to a likelihood-ratio test of that portfolio's efficiency, see Kandel and Stambaugh (1989).

The remainder of the paper is organized as follows. Section 2 shows that the cross sectional mean-beta relation fitted by OLS bears essentially no relation to the mean-variance location of the index portfolio. Section 3 defines a portfolio's relative efficiency, which can be

\footnotetext{
'Shanken's GLS estimator is defined using the covariance matrix of the residualo from the first-pass market-model regressions, but he shows in earlier work [Shanken (1985, footnote 16)] that the same eatimator is obtained using tbe covariance matrix of returns.
} 
stated in terms of either expected returns or variances, and section 4 provides simple relations between the index portfolio's relative efficiency and a GLS regression of expected returns on betas. Section 5 offers a portfolio-optimization setting in which to compare GLS to other methods for fitting and judging cross-sectional relations for expected returns. Conclusions are then presented in section 6. The Appendix contains proofs of all propositions.

\section{Inefficiency and Deviations from Mean-Beta Linearity}

For a universe of $n$ risky assets, define

$R: n$-vector of returns realized in a given period

$E: n$-vector of expected returns,

$V: n \times n$ covariance matrix of returns, assumed to be nonsingular.

For a given portfolio $p$, a combination of the $n$ assets, define

$w_{p}: n$-vector of weights in portfolio $p$,

$\mu_{p}$ : mean return on portfolio $p\left(=w_{p}^{\prime} E\right)$,

$\sigma_{p}^{2}$ : variance of return on portfolio $p\left(=w_{p}^{\prime} V w_{p}\right)$,

$\beta$ : $n$-vector of betas with respect to $p\left[=\left(1 / \sigma_{p}^{2}\right) V w_{p}\right]$.

Let $\iota$ denote an $n$-vector of ones, and define

$$
X=[\imath \beta] .
$$

Assume that neither $E$ nor $\beta$ are proportional to $\iota$.

The mean-variance location of portfolio $p$ has virtually no bearing on the degree to which the elements of $E$ and $\beta$ conform to a linear relation, when goodness-of-fit is measured by the standard Euclidean norm. That is, portfolio $p$ can lie arbitrarily close to the minimumvariance boundary and yet produce an OLS slope and R-squared that are arbitrarily close to zero. Similarly, portfolio $p$ can lie far from the minimum-variance boundary (by whatever metric desired) and yet still produce an OLS fit between expected returns and betas that is arbitrarily close to exact linearity.

We verify the above statements by considering "repackagings" of assets. The portfolio opportunities generated by one set of $n$ assets are identical to those generated by an alternative set of $n$ assets that simply repackage the original set, provided that returns on the new assets also have a nonsingular covariance matrix. Such a repackaging does not change 
the minimum-variance boundary or the location of portfolio $p$ in mean-variance space, but it can change the relation between the $n$ assets' expected returns and their betas with respect to portfolio $p$.

A given repackaging of assets can be represented by a nonsingular $n \times n$ matrix $A$, where $A \iota=\iota$. The returns on the repackaged assets are constructed as $R^{*}=A R$, so the means and betas of the repackaged assets are given by $E^{*}=A E$ and $\beta^{*}=A \beta$. For a given repackaging of the $n$ assets, let $\gamma^{*}$ denote the vector of coefficients in an ordinary-least-squares (OLS) regression of expected returns on betas with respect to portfolio $p$. That is,

$$
\gamma^{*}=\left(X^{*^{\prime}} X^{*}\right)^{-1} X^{*^{\prime}} E^{*}
$$

where

$$
X^{*}=\left[\iota \beta^{*}\right]=A X .
$$

The goodness of fit in this regression is given by

$$
R_{O L S}^{2}=1-\frac{\left(E^{*}-X^{*} \gamma^{*}\right)^{\prime}\left(E^{*}-X^{*} \gamma^{*}\right)}{\left(E^{*}-\frac{i^{\prime} E^{*}}{n} \iota\right)^{\prime}\left(E^{*}-\frac{i^{\prime} E^{*}}{n} \iota\right)} .
$$

If portfolio $p$ is inefficient, the following proposition states that one can always find a repackaging such that expected returns on the new set of $n$ assets obey essentially any desired OLS regression outcome.

Proposition 1 If portfolio $p$ is inefficient, then for any $\omega \in(0,1), \epsilon>0$, and two-element vector $\theta$, there exists a nonsingular $n \times n$ matrix $A$, with $A \iota=\iota$, such that ${ }^{3}$

$$
\begin{aligned}
\left\|\gamma^{*}-\theta\right\| & <\epsilon \text {, and } \\
R_{O L S}^{2} & =\omega .
\end{aligned}
$$

The results of an OLS regression correspond closely, of course, to what one would infer visually from a simple plot of expected returns versus betas. Proposition 1 implies that such a plot could in fact appear to contradict standard theory, since small degrees of portfolio inefficiency or deviations from perfect mean-beta linearity may not be visible in a plot. Two such examples are presented in Figure 1. The minimum variance boundaries in figures la and $1 c$ are identical, and they are generated using sample means and covariances of monthly

\footnotetext{
${ }^{3}$ The Euclidean norm of an $n$-vector $v$ is defined as $\|v\|=\left(v^{\prime} v\right)^{\frac{1}{t}}$ :
} 
returns on ten portfolios of common stocks sorted by equity capitalization (firm size) for the period from 1926-1992.

The ten points plotted in figure $1 \mathrm{a}$ as solid dots represent means and variances on ten assets that simply repackage the ten size portfolios. Portfolio $p$, shown as a small circle, is inefficient, having a monthly expected return that is 88 basis points less than the expected return on the efficient portfolio with the same variance. Figure $1 \mathrm{~b}$ plots the expected returns on the ten assets versus the assets' betas with respect to portfolio $p$. The mean-beta relation is not exactly linear, although the violations of exact linearity are too slight to be visible on the graph. The OLS regression line on which all of the points appear to lie has an intercept of 30 basis points, close to the average monthly interest rate for the $1926-92$ period, and the slope of the line is 76 basis points, the average excess return on portfolio $p$. In other words, shown only figure $1 b$, one would be inclined to conclude that portfolio $p$ is the Sharpe-Lintner tangent portfolio of the ten assets.

The ten assets whose means and variances are plotted in figure ic are obtained as a different repackaging of the ten size portfolios. Portfolio $q$ is inefficient, although it lies too close to the minimum-variance boundary for the inefficiency to be visible on the graph. For the ten assets, a least-squares regression of expected returns on betas with respect to portfolio $q$ produces an $R_{O L S}^{2}$ less than .0001 , and the corresponding plot is shown in figure Id. Shown only that plot, one would be inclined to conclude that portfolio $q$ is inefficient. Such a conclusion must be correct, of course, but the degree of inefficiency can be of no economic significance.

Related issues are discussed in several recent studies. Jagganathan and Wang (1993) construct a four-asset example in which repackaging changes $R_{O L S}^{2}$ from 0.95 to 0.0 , although those authors do not address the generality of the example or its relation to the mean-variance location of the index portfolio. The example in which $R_{O L S}^{2}$ is nearly zero is also related to the study by Roll and Ross (1994). They show that a portfolio can lie fairly close to the minimum-variance boundary and yet produce a mean-beta relation whose OLS slope is exactly zero (and thus $R_{O L S}^{2}$ is zero). Those authors consider a given set of $n$ assets, without repackaging, and they show that the region of zero-slope-producing portfolios moves closer to the minimum-variance boundary as the cross-sectional dispersion of expected returns on the $n$ assets grows smaller. As shown here, repackaging the assets allows the goodness of fit to become arbitrarily close to zero for any inefficient portfolio. Our observation that

\footnotetext{
${ }^{4}$ The portfolios include all stocks on the New York Stock Exchange, and the returns within a portfolio are value-weighted. Portfolio returns were obtained from the Index File supplied by the Cenler for Research in Security Prices.
} 
inefficient portfolios can also give arbitrarily good fits to any given linear mean-beta relation goes beyond the analysis of Roll and Ross, who do not consider goodness-of-fit measures." Grauer (1994) constructs a number of examples illustrating that the difference between the OLS intercept and the riskless rate does not correspond to the proximity of the index portfolio to the Sharpe-Linter tangent portfolio.

The plots in figure 1 omit much information about the covariance matrix of the assets. For example, the covariance matrix of the assets plotted in figure la, although nonsingular, has one very small eigenvalue. We consider below a framework that uses this additional information to measure the relation between expected returas and betas with quantities that correspond directly to portfolio $p$ 's position in mean-variance space. A portfolio's position in mean-variance space will be characterized by a simple measure of relative mean-variance efficiency.

\section{A Measure of Relative Portfolio Efficiency}

For a given portfolio $p$, let $x$ denote the efficient portfolio with the same variance as $p$, and let $y$ denote the minimum-variance portfolio with the same mean as $p$. Define

$\mu_{x}:$ mean return on portfolio $x$,

$\mu_{x_{0}}$ : mean return on portfolios uncorrelated with portfolio $x$,

$\sigma_{y}^{2}$ : variance of portfolio $y$.

$\mu_{g}$ : mean of the global minimum-variance portfolio,

$\sigma_{g}^{2}:$ global minimum variance.

The relative efficiency of portfolio $p$ is defined as

$$
\psi_{p} \equiv \frac{\mu_{p}-\mu_{g}}{\mu_{x}-\mu_{g}}
$$

The relative efficiency measure defined in (7) has a range from -1 to 1 , with the latter value corresponding to exact efficiency. Relative efficiency is undefined for the global minimumvariance portfolio. When portfolio $p$ lies on the minimum-variance boundary but has the lowest expected return for its variance, then $\psi_{p}=-1$. The square of this efficiency measure can also be expressed in terms of variances, as given by the following proposition.

\footnotetext{
${ }^{3}$ Roll and Ross derive mean-variance regions for portfolios that produce a given positive OLS slope for the mean-beta relation, but, other than in the case where the slope is zero, the value for the slope does not provide information about the goodness of fit.
} 


\section{Proposition 2}

$$
\psi_{p}^{2}=\frac{\sigma_{y}^{2}-\sigma_{g}^{2}}{\sigma_{p}^{2}-\sigma_{g}^{2}}
$$

Both (7) and (8) are represented graphically in figure 2. Figure 3 displays the locations in mean-variance space of portfolios with given values of $\psi_{p}$. The minimum-variance boundary is the same as that constructed in figures $l a$ and $l c$.

A portfolio's inefficiency can also be characterized in terms of correlation. Kandel and Stambaugh (1987) and Shanken (1987) show that $\rho_{p}$, the maximum correlation between the return on portfolio $p$ and the return on any minimum-variance portfolio, is given by

$$
\rho_{p}=\frac{\sigma_{y}}{\sigma_{p}}
$$

This measure, like $\psi_{p}$, approaches unity as portfolio $p$ approaches the minimum-variance boundary, but it is bounded below by zero. Combining (8) and (9) gives

$$
1-\rho_{p}^{2}=\left(1-\frac{\sigma_{g}^{2}}{\sigma_{p}^{2}}\right)\left(1-\psi_{p}^{2}\right)
$$

which implies that

$$
\psi_{p}<\rho_{p}
$$

if portfolio $p$ is inefficient.

\section{The Mean-Beta Relation and the Covariance Matrix}

Consider a cross-sectional regression of $E$ on $\beta$, where the covariance matrix $V$ is used to perform Generalized Least Squares (GLS). That is, the coefficient vector in the regression is given by

$$
\phi=\left[\begin{array}{l}
\phi_{1} \\
\phi_{2}
\end{array}\right]=\left(X^{\prime} V^{-1} X\right)^{-1} X^{\prime} V^{-1} E
$$

Proposition 3 The slope coefficient $\phi_{2}$ is given by

$$
\phi_{2}=\psi_{p}\left(\mu_{x}-\mu_{x 0}\right)
$$

and the intercept $\phi_{1}$ is given by $\phi_{1}=\mu_{p}-\phi_{2}$ or

$$
\phi_{1}=\mu_{x 0}+\left(1-\psi_{p}\right)\left(\mu_{s}-\mu_{x 0}\right)
$$


If $p$ is efficient, so $\psi_{p}=1$, then $\phi_{1}$ must equal $\mu_{x 0}$ and $\phi_{2}$ must be the portfolio's premium over that zero-beta rate, $\mu_{x}-\mu_{x 0}$. The above proposition reveals that, if $p$ is inefficient, then $\dot{\phi}_{1}>\mu_{x 0}$ and $\phi_{2}<\mu_{x}-\mu_{x 0}$. As $\psi_{p}$ gets closer to $1, \phi_{1}$ approaches $\mu_{x 0}$ and $\phi_{2}$ approaches its maximum value, $\mu_{x}-\mu_{x 0}$. A negative slope occurs for $\mu_{p}<\mu_{g}$, and a zero slope occurs if and only if $\psi_{p}=0$, or when $\mu_{p}=\mu_{g}{ }^{6}$

The standard measure for the GLS regression's goodness-of-fit is

$$
R_{G L S}^{2}=1-\frac{(E-X \phi)^{\prime} V^{-1}(E-X \phi)}{(E-\iota \bar{\mu})^{\prime} V^{-1}(E-\iota \bar{\mu})},
$$

where

$$
\bar{\mu}=\left(E^{\prime} V^{-1} \iota\right) /\left(\iota^{\prime} V^{-1} \iota\right),
$$

which is the coefficient in a GLS regression of $E$ on $t$. Note that exact linearity gives $R_{G L S}^{2}=1$, a slope of zero gives $R_{G L S}^{2}=0$, and $0 \leq R_{G L S}^{2} \leq 1$.

Proposition 4

$$
R_{G L S}^{2}=\psi_{p}^{2}
$$

We see that, unlike the OLS regression, the outcome of a GLS regression of expected returns on betas is determined completely by portfolio $p$ 's location in mean-variance space, as summarized by $\psi_{p}$. In figure $1 a, \psi_{p}=0.3$, so the goodness of fit in a GLS regression of means on betas with respect to portfolio $p$ is 0.09 . In figure $1 c, \psi_{q}$ is nearly 1 , and so is the goodness of fit in the GLS mean-beta regression. Although it can be shown algebraically that the coefficient vector $\phi$ and the goodness-of-fit measure $R_{G L S}^{2}$ are invariant to repackaging the $n$ assets, this result follows immediately from the fact that portfolio $p$ 's location in meanvariance space is unaffected by repackaging the assets used to generate the set of portfolio opportunities.

The GLS regression constructs a least-squares fit between means and betas that are transformed using the factored inverse of the covariance matrix, and, as is obvious from figure 1, the outcome of that regression need bear no resemblance to a plot of the "raw" expected returns versus betas. To decide whether fitted lines and goodness-of-fit measures are more relevant when computed with the raw means and betas than with their transformed counterparts, it may be useful to have a context in which fitted cross-sectional relations for expected returns would be used. The next section considers the use of such relations in providing expected returns as inputs to portfolio optimization. It is shown that the

\footnotetext{
${ }^{\circ}$ This last point is also made by Roll and Ross (1994), who attribute correspondence with Simon Wheatley.
} 
fitted GLS regression provides the optimal inputs for the optimization, and the regression's goodness-of-fit provides the squared relative efficiency of the resulting portfolio. If in other contexts the goodness-of-fit of the raw means and hetas is a more relevant metric, however, then one inust simply recognize that such a metric need hear no relation to the relative mean-variance efficiency of portfolio $p$.

\section{Using Fitted Mean Returns: An Optimization Setting}

In the absence of an exact linear relation between expected returns and betas, it seems useful to have an economic context in which one might, at a theoretical level, fit a linear relation and judge its goodness-of-fit. We consider here the simple context of mean-variance portfolio optimization, where the expected returns fitted from a linear cross-sectional relation are used as inputs to the problem of maximizing a portfolio's expected return for a given variance. The extent to which the portfolio constructed in the optimization differs from the efficient portfolio depends only on the differences between true and fitted expected returns.

Because the cross section of mean returns can possibly be explained better by variables used in addition to, or even in place of, betas computed against an inefficient portfolio, we allow such variables to be included in the analysis. For $k<n$, let $Z$ denote an $n \times k$ matrix of full column rank, where one column is $\iota$. The matrix $Z$ can simply be the $n \times 2$ matrix $X$ defined previously, so that the results below include fitting the mean-heta relation as a special case. We consider linear cross-sectional relations that fit expected returns as

$$
\hat{E}=Z a \text {. }
$$

for some $k \times 1$ vector $\alpha$.

The quality of the approximation to expected returns in (18) is characterized by the results of a portfolio optimization that uses $\hat{E}$ instead of $E$ as inputs. Let $w\left(\hat{E}_{;} \sigma^{2}\right)$ denote the solution to the portfolio maximization prohlem,

$$
\max _{w} w^{\prime} \hat{E}
$$

subject to the constraints

$$
\begin{aligned}
w^{\prime} V w & =\sigma^{2} \text { and } \\
w^{\prime} \iota & =1
\end{aligned}
$$

for a given $\sigma^{2}>\sigma_{g}^{2}$. 
Let $\delta$ denote the coefficient vector in a GLS regression of $E$ on $Z$,

$$
\delta=\left(Z^{\prime} V^{-1} Z\right)^{-1} Z^{\prime} V^{-1} E
$$

The fitted mean returns from the GLS regression are given by

$$
E^{\dagger}=Z \delta
$$

Note that $E^{\dagger}$ is a special case of $\hat{E}$ in (18) with $a=\delta$. We see that this choice of $a$ is best in the following sense.

\section{Proposition 5}

$$
\left[w\left(E^{\dagger} ; \sigma^{2}\right)\right]^{\prime} E \geq\left[w\left(\hat{E} ; \sigma^{2}\right)\right]^{\prime} E
$$

for all $a$.

In other words, the true expected return of the portfolio constructed using the GLS inputs is greater than or equal to the true expected return of a portfolio constructed using any other inputs of the form in (18).

As before, the goodness-of-fit for the GLS regression is given by

$$
R_{G L S}^{2}=1-\frac{(E-Z \delta)^{\prime} V^{-1}(E-Z \delta)}{(E-\iota \bar{\mu})^{\prime} V^{-1}(E-\iota \bar{\mu})},
$$

where $\bar{\mu}$ is defined as in (16). This goodness-of-fit is also the squared relative efficiency of the portfolio constructed using GLS inputs.

Proposition 6 For any $\sigma^{2}>\sigma_{g}^{2}$, let $q$ denote the portfolio with weights $w\left(E^{\dagger} ; \sigma^{2}\right)$. Then

$$
R_{G L S}^{2}=\psi_{q}^{2} .
$$

Note that $R_{G L S}^{2}$, and thus $\psi_{q}^{2}$, do not depend on the value for $\sigma^{2}$ specified in the portfolio optimization. In the special case where $Z=X$, it follows from propositions 4 and 6 that the portfolio constructed with the GLS inputs has the same (squared) relative efficiency as portfolio $p$. In [act, it can also be shown in that case that the weights in portfolio $p$ are equal to $w\left(E^{\dagger} ; \sigma_{p}^{2}\right)$. 


\section{Conclusions}

As is well known, an exact linear relation between expected returns and betas with respect to a given portfolio $p$ occurs if and only if portfolio $p$ lies exactly on the minimum-variance boundary. If portfolio $p$ is at all inefficient, however, a plot of expected returns versus betas bears essentially no relation to the position of portfolio $p$ in mean-variance space. An OLS slope and R-squared arbitrarily close to zero can occur when portfolio $p$ is arbitrarily close to the minumum-variance boundary. A near-perfect linear relation can occur, with any desired intercept and slope, if portfolio $p$ is grossly inefficient.

Although OLS is inadequate to the task, the exact linear mean-beta relation implied by the efficiency of portfolio $p$ can indeed be generalized to an approximate linear relation in the presence of inefficiency in portfolio $p$. If the linear relation is fitted as a GLS regression of expected returns on betas, using the variance-covariance matrix of returns, then that relation's coefficients and goodness-of-fit measure bear simple relations to the location of portfolio $p$ in mean-variance space. If portfolio $p$ is close to efficient, based on a relative efficiency measure that can be stated in terms of either means or variances, then the fitted relation will be close to the exact linear relation corresponding to an efficient portfolio whose mean and variance are close to those of portfolio $p$.

When portfolio $p$ is inefficient, it may be useful to adopt an economic context in which to fit a linear relation between expected return and beta and characterize, at a theoretical level, that relation's goodness-of-fit. We consider a context in which the quality of the linear relation is judged by its ability to provide fitted expected returns that are useful substitutes for true expected returns as inputs to a standard one-period portfolio optimization. For a given set of cross-sectional independent variables, including but not limited to beta, using the expected returns fitted from a GLS regression produces a portfolio with a higher expected return than using any other linear combination of the independent variables. The (squared) relative efficiency of that portfolio is simply the goodness-of-fit for the GLS regression.

The absence of a relation between the index portfolio's relative efficiency and a plot of expected returns versus betas illustrates the difficulty in using and assessing any model that delivers multiple implications. The Capital Asset Pricing Model, for example, delivers two major implications: (i) the market portfolio is mean-variance efficient and (ii) the relation between expected returns and betas is linear. Many finance academics prefer not to view these implications as separate, since either one implies the other, but such a strict view does not easily accommodate the fact that any financial model is at best a convenient and useful 
abstraction rather than an exact representation of reality. ${ }^{7}$ That is, the strict view does not easily entertain the possibility that, for practical purposes, one implication can hold while the other fajls. This study demonstrates that either implication can hold nearly perfectly while the other is grossly violated.

In some applications, the implication of interest may be that the market portfolio is mean-variance efficient or, in practical terms, very nearly so. This implication might lead, for example, to an "index fund" portfolio strategy. If the implication of interest is instead the cross-sectional mean-beta relation, then we see that the relative efficiency of the index portfolio offers little guidance as to the properties of such a relation. An additional problem with the mean-beta implication arises, however. Even if a linear mean-beta relation fits arbitrarily well (but not perfectly) for a given set of $N$ assets that generate all portfolio opportunities, the same relation can still provide a poor approximation for the expected return on another asset (a repackaging of the $N$ assets). Many applications of the model are likely to use a relation fitted with one set of assets to approximate the expected return on another asset, such as a project in a capital budgeting problem or a managed portfolio in a performance evaluation. Thus, unless one takes seriously the possibility that the linear meanbeta relation holds perfectly, this implication of the model seems to offer limited applicability.

\footnotetext{
'Such a view of modeling is advanced, for example, by Fama (1976).
} 


\section{Appendix}

Proof of Proposition 1: Let $F$ be a nonsingular $n \times n$ matrix whose first three columns are

$$
\begin{aligned}
& f_{1}=\imath, \\
& f_{2}=\beta,
\end{aligned}
$$

and

$$
f_{3}=E-X \theta,
$$

respectively. Note that when portfolio $p$ is inefficient, the above three vectors are linearly independent. Define the OLS coefficient vector

$$
\gamma=\left(X^{\prime} X\right)^{-1} X^{\prime} E
$$

Let $Q$ be an $n \times n$ diagonal matrix whose diagonal elements are all ones except for the $(3,3)$ element, which satisfies

$$
q_{(3,3)}<\frac{\epsilon}{\left(f_{3}^{\prime} X\left(X^{\prime} X\right)^{-2} X^{\prime} f_{3}\right)^{\frac{1}{2}}}
$$

when $\theta \neq \gamma$ and $q_{(3,3)}=1$ when $\theta=\gamma$. In the latter case, note that $f_{3}^{\prime} X=0$. Define the nonsingular matrix $B \equiv F Q F^{-1}$. It is easy to verify that the columns of $F$ are eigenvectors of $B$, and that the diagonal elements of $Q$ are the corresponding eigenvalues. Hence,

$$
\begin{aligned}
& B \iota=B f_{1}=f_{1} q_{(1,1)}=\imath, \\
& B \beta=B f_{2}=f_{2} q_{(2,2)}=\beta,
\end{aligned}
$$

and

$$
B f_{3}=f_{3} q_{(3,3)}
$$

Equations (A.6) and (A.7) can be rewritten as:

$$
B X=X \text {. }
$$

Let $G$ be a nonsingular $n \times n$ matrix whose columns are orthogonal to each other and whose first three columns are

$$
\begin{gathered}
g_{1}=\iota \\
g_{2}=\beta-\frac{\left(\iota^{\prime} \beta\right)}{n} \iota
\end{gathered}
$$

and

$$
g_{3}=B E-X\left(X^{\prime} X\right)^{-1} X^{\prime} B E
$$


Note that $g_{3}$ is the vector of residuals from the regression of $B E$ on $B X(=X)$. When portfolio $p$ is inefficient, the vector $E$ is not spanned by the columns of $X$, and, therefore, the above three columns of $G$ are linearly independent and orthogonal to each other. Let $\Lambda \equiv\left(G^{\prime} G\right)^{-1}$. Since the columns of $G$ are orthogonal to each other, $\Lambda$ is a diagonal matrix. Let $H$ be an $n \times n$ diagonal matrix whose diagonal elements are all ones except for the $(3,3)$ element, which is given by

$$
h_{(3,3)}=\left(\frac{\left(v^{\prime} v\right)(1-\omega)}{\left(g_{3}^{\prime} g_{3}\right) \omega}\right)^{\frac{1}{2}},
$$

where

$$
v \equiv X\left(X^{\prime} X\right)^{-1} X^{\prime} B E-\frac{\left(\iota^{\prime} B E\right)}{n} \iota .
$$

Define the nonsingular matrix $C \equiv G H \Lambda G^{\prime}$. It is easy to verify that the columns of $G$ are eigenvectors of $C$ as well as $C^{\prime}$, and the diagonal elements of $H$ are the corresponding eigenvalues of both $C$ and $C^{\prime}$. Hence,

$$
\begin{gathered}
C^{\prime} \iota=C \iota={ }^{\prime} g_{1}=g_{1} h_{(1,1)}=\iota, \\
C^{\prime} \beta=C \beta=C\left(g_{2}+\frac{\left(\iota^{\prime} \beta\right)}{n} g_{1}\right)=\beta, \\
C^{\prime} X=C X=X,
\end{gathered}
$$

and

$$
C g_{3}=h_{(3,3)} g_{3} \text {. }
$$

Now let $A \equiv C B$, which is nonsingular. Equations (A.9) and (A.17) imply that

$$
A X=C B X=C X=X,
$$

so $A \iota=\imath$. Substituting (A.19) into the definition of $\gamma^{*}$ in (2) and simplifying by using equations (A.3), (A.8), (A.9), and (A.17) gives:

$$
\begin{aligned}
\gamma^{-} & =\left(X^{\prime} A^{\prime} A X\right)^{-1} X^{\prime} A^{\prime} A E=\left(X^{\prime} X\right)^{-1} X^{\prime} C B E \\
& =\left(X^{\prime} X\right)^{-1} X^{\prime} B E=\left(X^{\prime} X\right)^{-1} X^{\prime} B\left(X \theta+f_{3}\right) \\
& =\theta+\left(X^{\prime} X\right)^{-1} X^{\prime} B f_{3} \\
& =\theta+q_{(3,3)}\left(X^{\prime} X\right)^{-1} X^{\prime} f_{3} .
\end{aligned}
$$

If $\gamma=\theta$, then $\gamma^{*}=\theta$, since in that case $q_{(3,3)}=1$ and $X^{\prime} f_{3}=0$. When $\gamma \neq \theta$, inequality (5) is obtained by combining (A.20) with (A.5):

$$
\left\|\gamma^{*}-\theta\right\|=\left(q_{(3,3)}^{2} f_{3}^{\prime} X\left(X^{\prime} X\right)^{-2} X^{\prime} f_{3}\right)^{\frac{1}{2}}<\epsilon
$$


Using (2), (3), (A.17), and (A.19) we get:

$$
\begin{aligned}
\left(E^{*}-X^{*} \gamma^{*}\right) & =A E-A X\left(X^{\prime} A^{\prime} A X\right)^{-1} X^{\prime} A^{\prime} A E \\
& =A E-X\left(X^{\prime} X\right)^{-1} X^{\prime} B E \\
& =C\left(B E-X\left(X^{\prime} X\right)^{-1} X^{\prime} B E\right) \\
& =C g_{3}=g_{3} h_{(3,3)}
\end{aligned}
$$

which implies that

$$
A E=g_{3} h_{(3,3)}+X\left(X^{\prime} X\right)^{-1} X^{\prime} B E .
$$

Using (A.17), (A.23) and the definition of $v$ in (A.14) we get:

$$
\begin{aligned}
E^{*}-\frac{\left(\iota^{\prime} E^{*}\right)}{n} \iota & =A E-\frac{\left(\iota^{\prime} A E\right)}{n} \iota \\
& =g_{3} h_{(3,3)}+X\left(X^{\prime} X\right)^{-1} X^{\prime} B E-\frac{\left(\iota^{\prime} B E\right)}{n} \iota \\
& =g_{3} h_{(3,3)}+v .
\end{aligned}
$$

Equation (6) is obtained by substituting (A.22) and (A.24) into (4), observing that $\left(v^{\prime} g_{3}\right)=0$, and using the definition of $h_{(3,3)}$ in (A.13):

$$
\begin{aligned}
R_{O L S}^{2} & =1-\frac{\left(E^{*}-X^{*} \gamma^{*}\right)^{\prime}\left(E^{*}-X^{*} \gamma^{*}\right)}{\left(E^{*}-\frac{\left(\iota^{\prime} E^{*}\right)}{n} \iota\right)^{\prime}\left(E^{*}-\frac{\left(\iota^{\prime} E^{*}\right)}{n} \iota\right)} \\
& =1-\frac{\left(g_{3}^{\prime} g_{3}\right) h_{(3,3)}^{2}}{\left(g_{3}^{\prime} g_{3}\right) h_{(3,3)}^{2}+v^{\prime} v} \\
& =\frac{v^{\prime} v}{\left(g_{3}^{\prime} g_{3}\right) h_{(3,3)}^{2}+v^{\prime} v} \\
& =\omega .
\end{aligned}
$$

Proof of Proposition 2: We first define the $2 \times 2$ matrix,

$$
\left[\begin{array}{cc}
L & M \\
M & N
\end{array}\right]=\left[\begin{array}{cc}
\iota^{\prime} V^{-1} \iota & \iota^{\prime} V^{-1} E \\
\iota^{\prime} V^{-1} E & E^{\prime} V^{-1} E
\end{array}\right]
$$

and its determinant,

$$
D=L N-M^{2} .
$$

As is well known [Roll (1977)],

$$
\begin{aligned}
& \mu_{g}=\frac{M}{L}, \\
& \sigma_{g}^{2}=\frac{1}{L}
\end{aligned}
$$


and if $\left(\mu, \sigma^{2}\right)$ is a point on the minimum variance boundary, then

$$
\sigma^{2}=\frac{\left(L \mu^{2}-2 M \mu+N\right)}{D} .
$$

Equation (A.30) can be rewritten as then:

$$
\sigma^{2}-\frac{1}{L}=\frac{L}{D}\left(\mu-\frac{M}{L}\right)^{2}
$$

By construction, $\left(\mu_{p}, \sigma_{y}^{2}\right)$ and $\left(\mu_{x}, \sigma_{p}^{2}\right)$ are on the minimum variance boundary. Using (A.28), (A.29), and (A.31) we get

$$
\begin{aligned}
\sigma_{y}^{2}-\sigma_{g}^{2} & =\frac{L}{D}\left(\mu_{p}-\mu_{g}\right)^{2}, \text { and } \\
\sigma_{p}^{2}-\sigma_{g}^{2} & =\frac{L}{D}\left(\mu_{x}-\mu_{g}\right)^{2} .
\end{aligned}
$$

Dividing equation (A.32) by (A.33) gives

$$
\left(\frac{\mu_{p}-\mu_{g}}{\mu_{x}-\mu_{g}}\right)^{2}=\left(\frac{\sigma_{y}^{2}-\sigma_{g}^{2}}{\sigma_{p}^{2}-\sigma_{g}^{2}}\right)
$$

Equation (8) follows from (A.34) and the definition of $\psi$ in (7).

Proof of Proposition 3: The geometric analysis of the GLS coefficients in Roll (1985) may be used as a starting point for this proof. For the sake of clarity, we provide a complete proof. Observe that

$$
X=\left[\iota \frac{1}{w_{p}^{\prime} V w_{p}} V w_{p}\right]
$$

so

$$
\begin{gathered}
X^{\prime} V^{-1} X=\left[\begin{array}{cc}
\iota^{\prime} V^{-1} \iota & \frac{1}{w_{p}^{\prime} V w_{p}} \\
\frac{1}{w_{p}^{\prime} V w_{p}} & \frac{1}{w_{p}^{\prime} V w_{p}}
\end{array}\right]=\frac{1}{\sigma_{p}^{2}}\left[\begin{array}{cc}
L \sigma_{p}^{2} & 1 \\
1 & 1
\end{array}\right], \\
\left(X^{\prime} V^{-1} X\right)^{-1}=\frac{\sigma_{p}^{2}}{L \sigma_{p}^{2}-1}\left[\begin{array}{cc}
1 & -1 \\
-1 & L \sigma_{p}^{2}
\end{array}\right],
\end{gathered}
$$

and

$$
X^{\prime} V^{-1} E=\left[\begin{array}{c}
\iota^{\prime} V^{-1} E \\
\frac{w_{p}^{\prime} E}{w_{p}^{\prime} V w_{p}}
\end{array}\right]=\left[\begin{array}{c}
M \\
\frac{\mu_{p}}{\sigma_{p}^{2}}
\end{array}\right] \text {. }
$$

Multiplying (A.37) and (A.38) gives

$$
\phi=\frac{\sigma_{p}^{2}}{L \sigma_{p}^{2}-1}\left[\begin{array}{c}
M-\frac{\mu_{p}}{\sigma_{p}^{2}} \\
L \mu_{p}-M
\end{array}\right] .
$$

Using (A.28) and (A.29), the second element of $\phi$ in (A.39) can be written as

$$
\phi_{2}=\left(\frac{\sigma_{p}^{2}}{\sigma_{p}^{2}-\sigma_{g}^{2}}\right)\left(\mu_{p}-\mu_{g}\right) .
$$


This expression for $\phi_{2}$ is presented also by Roll (1985). The expression in (13) is obtained by observing that, since portfolio $x$ is on the minimum-variance boundary, we can write $\mu_{o}$ as

$$
\begin{aligned}
\mu_{g} & =\mu_{x 0}+\left(\mu_{x}-\mu_{x 0}\right) \frac{\operatorname{cov}\left\{r_{g}, r_{x}\right\}}{\sigma_{x}^{2}} \\
& =\mu_{x 0}+\left(\mu_{x}-\mu_{x 0}\right) \frac{\sigma_{g}^{2}}{\sigma_{p}^{2}},
\end{aligned}
$$

where the second line follows by substituting $\sigma_{p}^{2}$ for $\sigma_{x}^{2}$ (equal by construction) and from the property that every asset has covariance $\sigma_{g}^{2}$ with the global minimum-variance portfolio [Roll (1977)]. Equation (A.41) can be rewritten as

$$
\sigma_{p}^{2}=\sigma_{g}^{2} \frac{\left(\mu_{x}-\mu_{x 0}\right)}{\left(\mu_{g}-\mu_{x 0}\right)}
$$

Substituting (A.42) for $\sigma_{p}^{2}$ in (A.40), simplifying, and using the definition of $\psi_{p}$ in (7) gives (13). The expression for $\phi_{1}$ in (14) follows directly by substituting (A.28), (A.29) and (A.41) into the first element of $\phi$ in (A.39) and simplifying.

Proof of Proposition 4: Equation (15) can be rewritten as:

$$
1-R_{G L S}^{2}=\frac{(E-X \phi)^{\prime} V^{-1}(E-X \phi)}{(E-\iota \bar{\mu})^{\prime} V^{-1}(E-\iota \bar{\mu})},
$$

Through straightforward algebra, using (A.26)-(A.39), one can express the numerator of $\left(1-R_{G L S}^{2}\right)$ in $(\mathrm{A.43)}$ as

$$
\begin{aligned}
(E-X \phi)^{\prime} V^{-1}(E-X \phi) & =\frac{L \mu_{p}^{2}-2 M \mu_{p}+N-D \sigma_{p}^{2}}{1-L \sigma_{p}^{2}} \\
& =\frac{D}{L} \frac{\sigma_{p}^{2}-\sigma_{y}^{2}}{\sigma_{p}^{2}-\sigma_{g}^{2}}
\end{aligned}
$$

where the second line makes use of the equation for the minimum-variance boundary (A.30),

$$
\sigma_{v}^{2}=\left(L \mu_{p}^{2}-2 M \mu_{p}+N\right) / D
$$

The denominator of $\left(1-R_{G L S}^{2}\right)$ can be expressed, using (16), (A.26), and (A.27), as

$$
(E-\iota \bar{\mu})^{\prime} V^{-1}(E-\iota \bar{\mu})=\frac{D}{L} .
$$

Taking 1 minus the ratio of (A.44) to (A.46) gives

$$
R_{G L S}^{2}=\frac{\left(\sigma_{y}^{2}-\sigma_{g}^{2}\right)}{\left(\sigma_{p}^{2}-\sigma_{g}^{2}\right)}
$$


which is equal to $\psi_{p}^{2}$ using Proposition 2.

Proof of Proposition 5: Since $w\left(\dot{E} ; \sigma^{2}\right)$ is the solution to the portfolio maximization problem (19)-(21), there exist scalars $\zeta_{1} \geq 0$ and $\zeta_{2}$ such that the following first order condition is satisfied:

$$
\begin{aligned}
w\left(\hat{E} ; \sigma^{2}\right) & =\zeta_{1} V^{-1} \hat{E}+\zeta_{2} V^{-1} \iota \\
& =\zeta_{1} V^{-1} Z a+\zeta_{2} V^{-1} \iota
\end{aligned}
$$

where the second line uses (18). The maximization problem's constraints imply that

$$
\begin{aligned}
\zeta_{2} & =\frac{1}{L}-\zeta_{1} \iota^{\prime} V^{-1} Z a, \text { and } \\
\zeta_{1} & =\left(\frac{L \sigma^{2}-1}{L a^{\prime} Z^{\prime} V^{-1} Z a-\left(\iota^{\prime} V^{-1} Z a\right)^{2}}\right)^{\frac{1}{2}} .
\end{aligned}
$$

Using (A.26) and (A.49), the expected return $\left[w\left(\hat{E} ; \sigma^{2}\right)\right]^{\prime} E$ can be written as

$$
\left[w\left(\hat{E} ; \sigma^{2}\right)\right]^{\prime} E=\frac{M}{L}+\zeta_{1}\left[\left(E^{\prime} V^{-1} Z a\right)-\frac{M}{L}\left(\iota^{\prime} V^{-1} Z a\right)\right] .
$$

Recall that $\delta=\left(Z^{\prime} V^{-1} Z\right)^{-1} Z^{\prime} V^{-1} E$, define

$$
K \equiv E^{\prime} V^{-1} Z \delta=\left(E^{\prime} V^{-1} Z\right)\left(Z^{\prime} V^{-1} Z\right)^{-1}\left(Z^{\prime} V^{-1} E\right),
$$

and note that $K \geq\left(M^{2} / L\right)$. Let $d_{1}$ be an $n$-vector with 1 in the first element and 0 elsewhere. Noting that the first column of $Z$ is $\iota$, it is easily verified that

$$
\begin{gathered}
Z d_{1}=\iota, \\
d_{1}=\left(Z^{\prime} V^{-1} Z\right)^{-1} Z^{\prime} V^{-1} \iota, \\
\delta^{\prime} Z^{\prime} V^{-1} \iota=E^{\prime} V^{-1} Z d_{1}=E^{\prime} V^{-1} \iota=M, \text { and } \\
\iota^{\prime} V^{-1} Z d_{1}=d_{1}^{\prime} Z^{\prime} V^{-1} Z d_{1}=\iota^{\prime} V^{-1} \iota=L .
\end{gathered}
$$

For any $n$-vector $a$ there exist scalars $c_{1}$ and $c_{2}$ and an $n$-vector $u$ such that

$$
\begin{aligned}
a & =c_{1} \delta+c_{2} d_{1}+u, \\
u^{\prime}\left(Z^{\prime} V^{-1} Z\right) d_{1} & =u^{\prime} Z^{\prime} V^{-1} \iota=0, \text { and } \\
u^{\prime}\left(Z^{\prime} V^{-1} Z\right) \delta & =u^{\prime} Z^{\prime} V^{-1} E=0 .
\end{aligned}
$$

Maximizing the expected return $\left[w\left(\hat{E} ; \sigma^{2}\right)\right]^{\prime} E$ with respect to $a$ is, therefore, equivalent to maximizing the expected return with respect to $c_{1}, c_{2}$, and an $n$-vector $u$ that satisfies (A.58) 
and (A.59). Using (A.55), (A.56), (A.58), and (A.59), we get

$$
\begin{aligned}
\iota^{\prime} V^{-1} Z a & =c_{1}\left(\iota^{\prime} V^{-1} Z \delta\right)+c_{2}\left(\iota^{\prime} V^{-1} Z d_{1}\right)+\left(\iota^{\prime} V^{-1} Z u\right) \\
& =c_{1} M+c_{2} L, \\
E^{\prime} V^{-1} Z a & =c_{1}\left(E^{\prime} V^{-1} Z \delta\right)+c_{2}\left(E^{\prime} V^{-1} Z d_{1}\right)+\left(E^{\prime} V^{-1} Z u\right) \\
& =c_{1} K+c_{2} M, \text { and } \\
a^{\prime} Z^{\prime} V^{-1} Z a & =c_{1}{ }^{2} K+c_{2}{ }^{2} L+2 c_{1} c_{2} M+u^{\prime} Z^{\prime} V^{-1} Z u
\end{aligned}
$$

Substituting (A.60)-(A.62) into (A.50), and (A.51) gives

$$
\left[w\left(\hat{E}_{;} \sigma^{2}\right)\right]^{\prime} E=\frac{M}{L}+\frac{\left(L \sigma^{2}-1\right)^{\frac{1}{2}} c_{1}\left(K L-M^{2}\right)}{\left[c_{1}^{2}\left(K L-M^{2}\right)+\left(u^{\prime} Z^{\prime} V^{-1} Z_{u}\right)\right]^{\frac{1}{2}} L} .
$$

Let $c_{2}=0$, since (A.63) does not depend on $c_{2}$. The maximum must occur with $c_{1}>0$ since the denominator and the other factors in the numerator are positive. For any $c_{1}>0$, the maximum occurs at $u=0$ and does not depend on $c_{1}$. Thus, let $c_{1}=1$, which implies the maximum occurs at $a=\delta$ or $\hat{E}=Z \delta=E^{\dagger}$.

Proof of Proposition 6: We first observe that $(E-Z \delta)$ can be written as

$$
(E-Z \delta)=\left[I-Z\left(Z^{\prime} V^{-1} Z\right)^{-1} Z^{\prime} V^{-1}\right] E,
$$

which, when substituted into the numerator of $\left(1-R_{Z}^{2}\right)$ in (A.43), provides

$$
\begin{aligned}
& (E-Z \delta)^{\prime} V^{-1}(E-Z \delta) \\
& =E^{\prime}\left[I-Z\left(Z^{\prime} V^{-1} Z\right)^{-1} Z^{\prime} V^{-1}\right]^{\prime} V^{-1}\left[I-Z\left(Z^{\prime} V^{-1} Z\right)^{-1} Z^{\prime} V^{-1}\right] E \\
& =E^{\prime} V^{-1} E-E^{\prime} V^{-1} Z\left(Z^{\prime} V^{-1} Z\right)^{-1} Z^{\prime} V^{-1} E \\
& =N-K
\end{aligned}
$$

where the last line uses (A.26) and (A.52). The denominator of $1-R_{G L S}^{2}$ can be expressed, using (16), (A.26), and (A.27), as

$$
(E-\iota \bar{\mu})^{\prime} V^{-1}(E-\iota \bar{\mu})=\frac{D}{L} .
$$

Taking 1 minus the ratio of (A.66) to (A.67) and using (A.27) gives

$$
R_{G L S}^{2}=\frac{\left(K L-M^{2}\right)}{D}
$$

Let $\mu_{q}$ and $\sigma_{q}^{2}$ denote the mean and variance of the return on the portfolio $q$, respectively. By Proposition 1,

$$
\psi_{q}^{2}=\frac{\sigma_{y}^{2}-\sigma_{g}^{2}}{\sigma_{q}^{2}-\sigma_{g}^{2}}
$$


where $\sigma_{y}^{2}$ denotes here the variance of the minimum variance portfolio with mean return $\mu_{q}$. Equation (A.32) implies that the numerator of (A.69) can be written as

$$
\sigma_{y}^{2}-\sigma_{g}^{2}=\frac{L}{D}\left(\mu_{q}-\mu_{g}\right)^{2}
$$

Substituting $\mu_{q}, \sigma_{q}^{2}$, and $\mu_{g}$ into (A.63) gives:

$$
\left(\mu_{q}-\mu_{g}\right)^{2}=\frac{\left(\sigma_{q}^{2}-\sigma_{g}^{2}\right)\left(K L-M^{2}\right)}{L}
$$

which implies that the denominator of (A.69) can be written as:

$$
\sigma_{q}^{2}-\sigma_{g}^{2}=\frac{L\left(\mu_{q}-\mu_{g}\right)^{2}}{K L-M^{2}}
$$

Dividing (A.70) by (A.72) yields

$$
\psi_{q}^{2}=\frac{\left(K L-M^{2}\right)}{D}=R_{G L S}^{2}
$$

where the second equality is based on (A.68). 
Fig. 1a

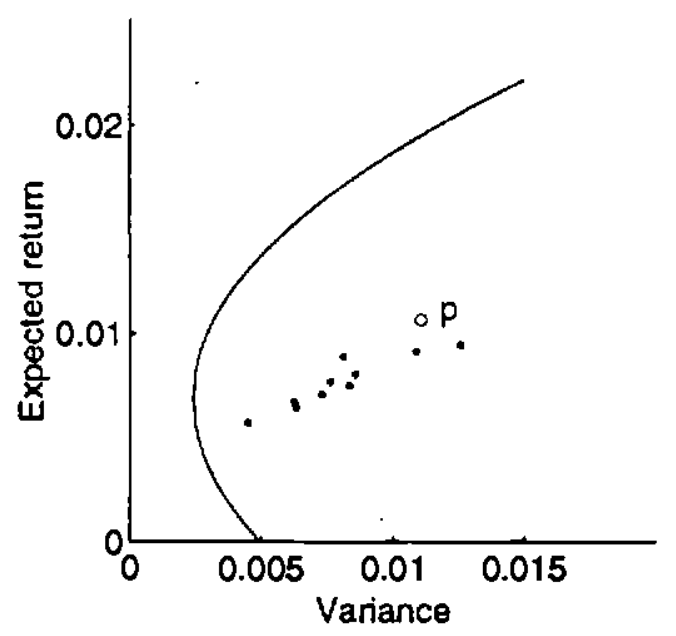

Fig. 1c

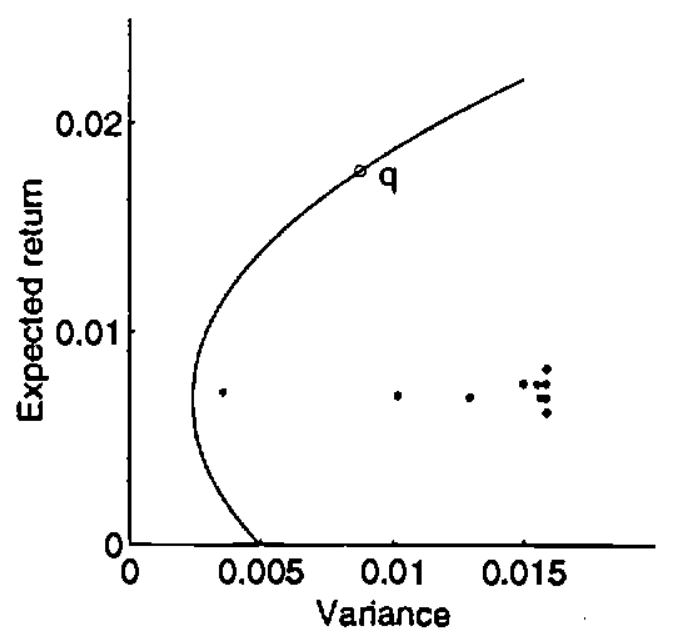

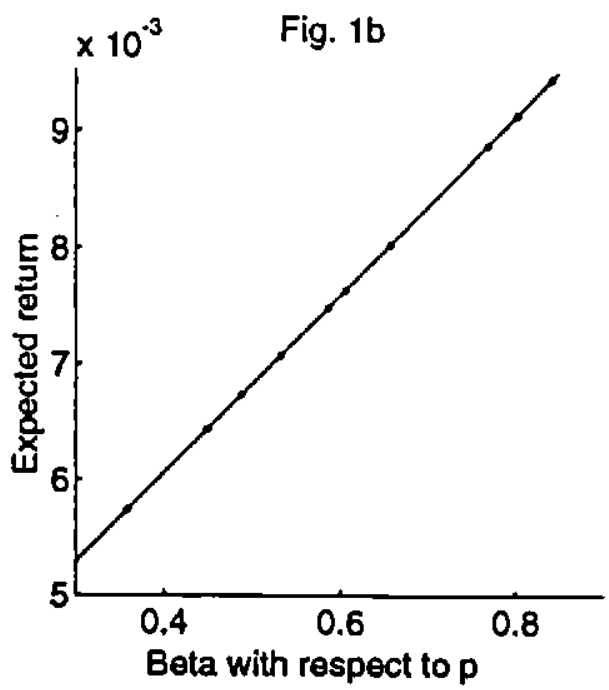

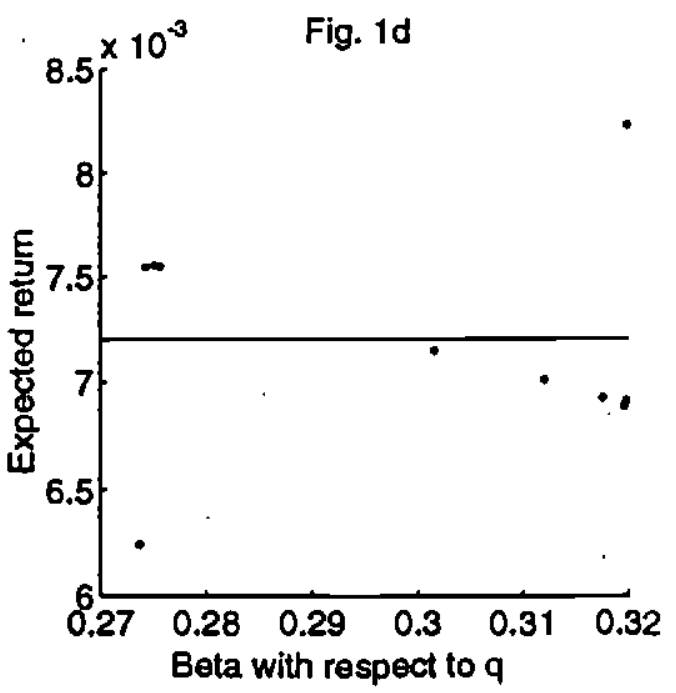

Figure 1. Examples of mean-beta relations for different mean-variance locations of the index portfolio. Figure la plots ten assets (solid dots), their minimumvariance boundary, and a portfolio $p$ of those ten assets (circle). Figure $1 \mathrm{~b}$ plots the expected returns and betas of those assets with respect to portfolio $p$ as well as the OLS regression line through those points. Figures $\mathrm{lc}$ and $1 \mathrm{~d}$ display a similar case, except that the ten assets are a "repackaging" of those in the first case. The points in figure $1 \mathrm{~b}$ do not lie exactly on the regression line, and portfolio $g$ in figure $1 \mathrm{c}$ does not lie exactly on the minimum-variance boundary. 


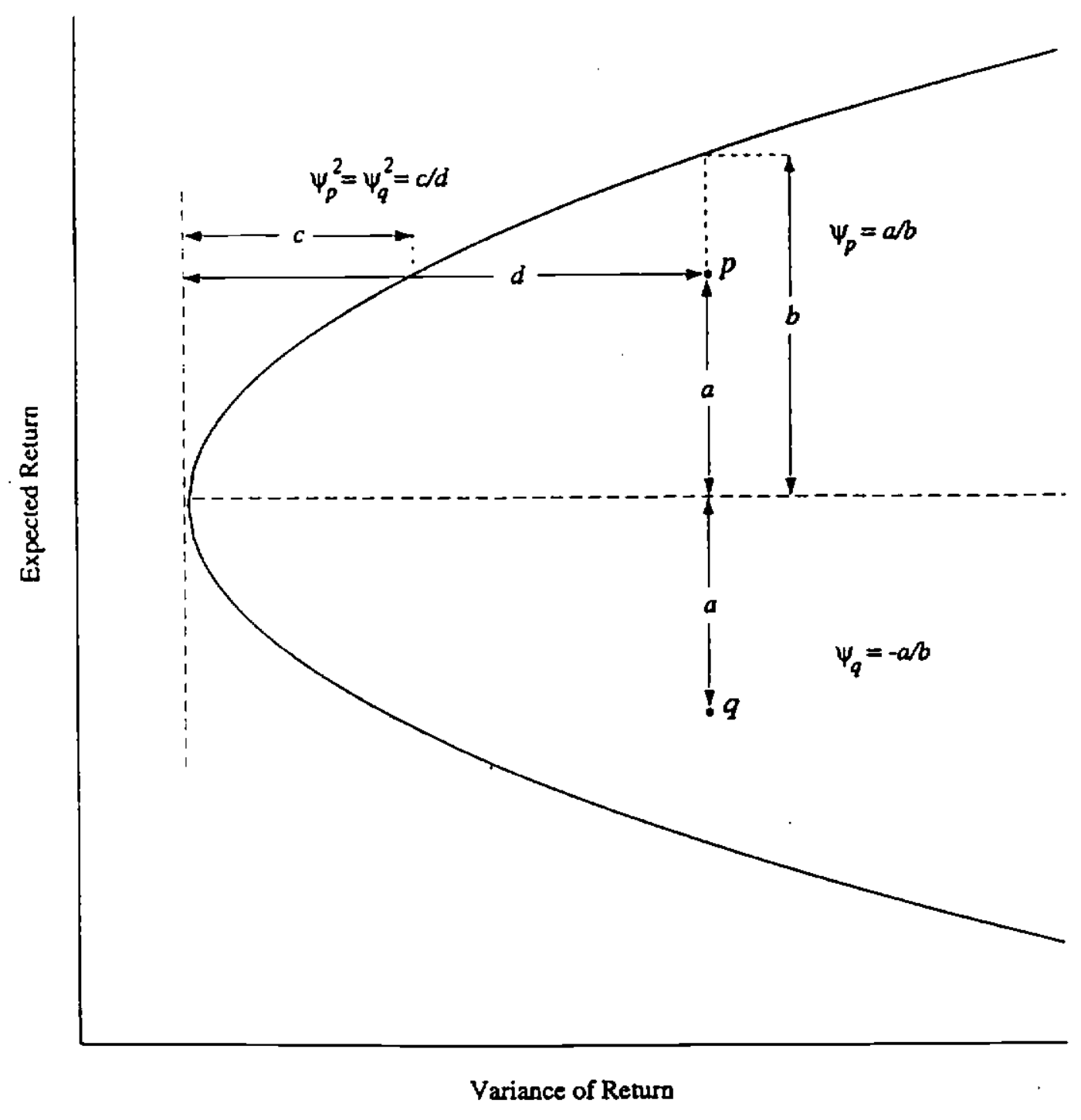

Figure 2. The inefficiency measure $\psi$ in mean-variance space. 


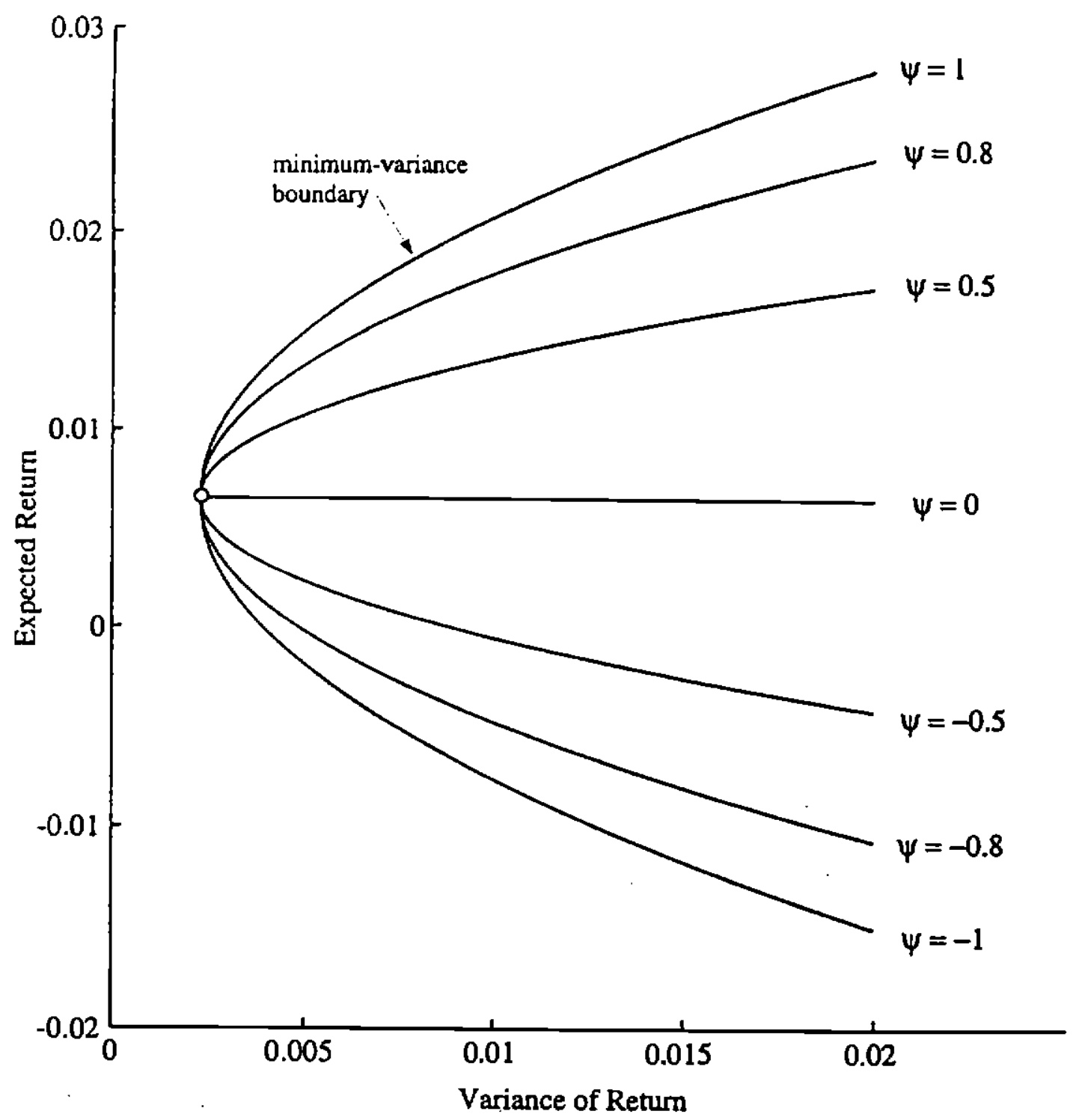

Figure 3. Mean-variance locations of portfolios with various levels of relative efficiency $\psi$. Relative efficiency is undefined for the global minimum-variance portfolio. 


\section{References}

Amihud, Yakov, Bent Jesper Christensen, and Haim Mendelson, 1992, "Further Evidence on the Risk-Return Relationship," working paper, New York University and Stanford University.

Fama, Eugene F., 1976, Foundations of Finance, Basic Books, New York.

Fama, Eugene F. and Kenneth R. French, 1992, "The Cross-Section of Expected Stock Returns," Journal of Finance 47, 427-465.

Grauer, Robert R., 1994, "Tests of the Capital Asset Pricing Model Based on the CrossSection of Expected Returns, ${ }^{n}$ working paper, Simon Fraser University.

Jagannathan, Ravi and Zhenyu Wang, 1993, "The CAPM is Alive and Well," working paper, University of Minnesota.

Kandel, Shmuel and Robert F. Stambaugh, 1987, "On Correlations and Inferences about Mean-Variance Efficiency," Journal of Financial Economics 18, 61-90.

Kandel, Shmuel and Robert F. Stambaugh, 1989, "A Mean-Variance Framework for Tests of Asset Pricing Models, ${ }^{n}$ Review of Financial Studies 2, 125-156.

Roll, Richard, 1977, "A Critique of the Asset Pricing Theory's Tests; Part 1: On Past and Potential Testability of the Theory," Journal of Financial Economics 4, 129-176.

Roll, Richard, 1985, "A Note on the Geometry of Shanken's CSR T ${ }^{2}$ Test for Mean/Variance Efficiency, ${ }^{n}$ Journal of Financial Economics 14, 349-357.

Roll, Richard, and Stephen A. Ross, 1994, "On the Cross-Sectional Relation Between Expected Returns and Betas," Journal of Finance, forthcoming.

Ross, Stephen A., 1977, "The Capital Asset Pricing Model (CAPM), Short-Sale Restrictions and Related Issues," Journal of Finance 32, 177-183.

Shanken, Jay, 1985, "Multivariate Tests of the Zero-Beta CAPM," Journal of Financial Economics 14, 327-348.

Shanken, Jay, 1987, “Multivariate Proxies and Asset Pricing Relations: Living with the Roll Critique," Journal of Financial Economics 18, 91-110.

Shanken, Jay, 1992, "On the Estimation of Beta-Pricing Models," The Review of Financial Studies 5, 1-33. 